\title{
New Complexity Results for the $k$-Covers Problem
}

\author{
Costas S. Iliopoulos * $\quad$ Manal Mohamed ${ }^{\dagger} \quad$ W.F. Smyth ${ }^{\ddagger}$
}

\begin{abstract}
The $k$-covers problem $(k \mathrm{CP})$ asks us to compute a minimum cardinality set of strings of given length $k>1$ that covers a given string. It was shown in a recent paper, by reduction to 3 -SAT, that the $k$-covers problem is NP-complete. In this paper we introduce a new problem, that we call the $k$-Bounded Relaxed Vertex Cover Problem $\left(\mathrm{RVCP}_{k}\right)$, which we show is equivalent to $k$-Bounded Set Cover $\left(\mathrm{SCP}_{k}\right)$. We show further that $k \mathrm{CP}$ is a special case of $\mathrm{RVCP}_{k}$ restricted to certain classes $G_{\boldsymbol{x}, k}$ of graphs that represent all strings $\boldsymbol{x}$. Thus a minimum $k$-cover can be approximated to within a factor $k$ in polynomial time. We discuss approximate solutions of $k \mathrm{CP}$, and we state a number of conjectures and open problems related to $k \mathrm{CP}$ and $G_{\boldsymbol{x}, k}$.
\end{abstract}

Keywords: string, cover, regularity, complexity, NP-complete.

\section{Introduction}

The computation of various kinds of "regularities" in given strings $\boldsymbol{x}=\boldsymbol{x}[1 . . n]$ has been of interest for a quarter-century, signalled by the publication in the early 1980s of several $O(n \log n)$-time algorithms for computing all repetitions (adjacent identical substrings) $[7,3,16]$, work that has more recently been refined to $O(n)$-time algorithms $[15,13]$. In response to applications arising in data compression and molecular biology, the computation of repetitions was generalized to computation of repeats (adjacency condition dropped), for which also $O(n)$-time algorithms have been found [5, 8]; then still further to computation of approximate repeats [17].

In [2] the idea of a quasiperiod or cover was introduced; that is, a proper substring $\boldsymbol{u}$ of the given string $\boldsymbol{x}$ such that every position of $\boldsymbol{x}$ is contained in an occurrence of $\boldsymbol{u}$. Several algorithms to compute covers of $\boldsymbol{x}$ were published in the 1990s, culminating in an algorithm [14] that in $O(n)$ time computes a cover array specifying all the covers (quasiperiods) of every prefix of $\boldsymbol{x}$; this algorithm thus directly generalizes the border array ("failure function") algorithm [1] that specifies all the borders, hence all the periods, of every prefix of $\boldsymbol{x}$.

In [12] a further extension, the $k$-covers problem, was introduced: compute a minimum set $U_{\nu}=\left\{\boldsymbol{u}_{1}, \boldsymbol{u}_{2}, \ldots, \boldsymbol{u}_{\boldsymbol{\nu}}\right\}$ of strings of given length $k>1$ such that every position of $\boldsymbol{x}$ is contained in an occurrence of some element of $U_{\nu}$. A polynomial-time algorithm was given

\footnotetext{
${ }^{*}$ Department of Computer Science, King's College London, London WC2R 2LS, England; csi@dcs.kcl.ac.uk.

${ }^{\dagger}$ Department of Computer Science, King's College London, London WC2R 2LS, England; manal@dcs.kcl.ac.uk.

${ }^{\ddagger}$ Algorithms Research Group, Department of Computing \& Software, McMaster University, Hamilton, Ontario, Canada L8S 4K1; Department of Computing, Curtin University, Perth WA 6845, Australia; smyth@computing.edu.au.
} 
for this problem, later discovered to be incorrect [18]; just recently the problem itself has been shown to be NP-complete, based on a reduction to 3-SAT [6]. In this latter paper, two $O(n \log n)$ algorithms were described that yielded an approximation to a minimum $k$-cover of $\boldsymbol{x}$; it was conjectured that these algorithms would yield a $k$-cover of cardinality at most $\log n$ times the minimum.

In Section 2 of this paper we introduce a new NP-complete problem which we call the relaxed vertex cover problem. We show that a special case of this problem is equivalent to the $k$-bounded set cover problem. We call this subproblem $k$-bounded relaxed vertex cover $\left(\mathrm{RVCP}_{k}\right)$.

In Section 3 we show that the $k$-covers problem is a subproblem of $\mathrm{RVCP}_{k}$. Thus the existence of an approximation algorithm that achieves at least a ratio of $k$ times the minimum is assured. The new reduction of $k$-covers raises the possibility that in fact $k$ covers can also be approximated to within a lower factor.

In Section 4 we discuss conjectures and open problems derived from the complexity analysis of the $k$-covers problem, both here and in [6].

\section{The Relaxed Vertex Cover Problem}

In this section, we introduce a new problem which we call the relaxed vertex cover problem. Given a directed graph $G=(V, E)$, where $V_{o} \subseteq V$ is the set of all vertices in $V$ with out-degree $>0$, find the smallest subset $V^{\prime} \subseteq V_{o}$ such that if $(u, v) \in E$, then one of the following conditions holds:

(C1) $u \in V^{\prime}$;

(C2) $v \in V^{\prime}$;

(C3) there exist $w_{u}, w_{v} \in V^{\prime}$ such that $\left(w_{u}, u\right) \in E$ and $\left(w_{v}, v\right) \in E$.

We say that $V^{\prime}$ is a vertex semi-cover of $G$.

The decision form of the relaxed vertex cover problem asks for given $G$ and $\nu$, whether there exists a vertex semi-cover $V^{\prime} \subseteq V_{o}$ of $G$ such that $\left|V^{\prime}\right|=\nu$. We call this problem $\boldsymbol{R V} \boldsymbol{C P}$. If the in-degree of all vertices in $V-V_{o}$ is no more than $k$ we call this problem the $k$-bounded relaxed vertex cover problem $\left(\boldsymbol{R} \boldsymbol{V} \boldsymbol{C} \boldsymbol{P}_{k}\right)$ and we show that it is equivalent to the $k$-bounded set cover problem $\left(\mathrm{SCP}_{k}\right)$.

$\mathrm{SCP}_{k}$ is a special case of the set cover problem and is defined as follows: given a collection $U$ of subsets of a finite set $S$ where the number of occurrences in $U$ of any element is bounded by a constant $k$, find a minimum size subset $U^{\prime} \subseteq U$ such that every element in $S$ belongs to at least one member in $U^{\prime}$. This problem is well-known to be NP-complete [9]. Bar-Yehuda and Even [4], and Hochbaum [11] presented polynomial time $k$-approximation algorithms for this problem. Halperin [10] described the most effective such algorithm which yields a subset whose cardinality is $k-\frac{(k-1) \cdot \ln \ln n}{\ln n}$ times the minimum.

Theorem 1 Problem $R V C P_{k}$ is equivalent to $S C P_{k}$.

Proof: First, we show that $\mathrm{RVCP}_{k}$ can be reduced to $\mathrm{SCP}_{k}$ in polynomial time. Suppose we are given a directed graph $G=(V, E)$ together with a subset $V^{\prime}$ of $V_{o}$, where $V_{o} \subseteq V$ is the set of all vertices with out-degree $>0$, an instance of $\mathrm{RVCP}_{k}$. We construct a set $S$ from $E$ and a collection $U$ of subsets of $S$, an instance of $\mathrm{SCP}_{k}$. Then we show how $V^{\prime}$ can 
be used to calculate a set cover $U^{\prime}$ such that $U^{\prime}$ is a cover of $S$ if and only if $V^{\prime}$ is a vertex semi-cover of $G$.

Suppose the vertices of $V$ are labelled $1,2, \ldots, n$ and the arcs $(u, v)$ are labelled $u v$. Let $S$ be the set of labels of arcs of $E$. The set $U$ (initially empty) is constructed as follows: for each vertex $v \in V_{o}$,

1. Determine $N(v)=\{i \mid(v, i) \in E\}$, the set of vertices adjacent to vertex $v$ (outneighbors of $v$ ).

2. Form $O_{v}=\{v u \mid(v, u) \in E\}$, the set of the outgoing arcs.

3. Form $I_{v}=\{u v \mid(u, v) \in E\}$, the set of incoming arcs.

4. Form $C_{v}=\{u w \mid(u, w) \in E ; u, w \in N(v)\}$. the set of arcs between the out-neighbors of $v$.

5. Form $U_{v}=I_{v} \cup O_{v} \cup C_{v}$.

6. Update $U \leftarrow U \cup\left\{U_{v}\right\}$.

Note that each set $U_{v}$ corresponds to the set of arcs that could be semi-covered by vertex $v$. The sets $C_{v}$ are the sets of arcs that satisfy condition (C3). It is not difficult to see that each arc $\left(v_{1}, v_{2}\right)$, where $v_{1}, v_{2} \in V_{o}$, appears exactly twice in $U$, while the rest of the arcs cannot appear more than $k$ times. This is because the in-degree of each vertex in $V-V_{o}$ is no more than $k$.

By construction, we see that $V^{\prime}=\left\{i_{1}, i_{2}, \ldots, i_{\left|V^{\prime}\right|}\right\}$ is a semi-cover of $G$ if and only if the corresponding set $U^{\prime}=\left\{U_{i_{1}}, U_{i_{2}}, \ldots, U_{i_{\left|V^{\prime}\right|}}\right\}$ is a cover of $S$.

Second, we show that $\mathrm{SCP}_{k}$ can also be reduced to $\mathrm{RVCP}_{k}$ in polynomial time. Let $S=\left\{e_{1}, e_{2}, \ldots e_{|S|}\right\}$ and $U=\left\{U_{1}, U_{2}, \ldots, U_{|U|}\right\}$ be a given instance of $\mathrm{SCP}_{k}$. We construct a graph $G=(V, E)$ such that $|V|=|S|+|U|$, where $|S|$ vertices are associated with the elements in $S$ (element-vertices) and $|U|$ vertices are associated with the distinct subsets in $U$ (subset-vertices). The set of $\operatorname{arcs} E$ is constructed by adding an $\operatorname{arc}(u, v)$ from each subset-vertex $u$ to each element-vertex $v$ that belongs to the subset represented by $u$. Additional arcs are added between the subset-vertices if the two subsets share one or more elements. More formally $E$ is constructed according to the following steps, each performed for every element $U_{i} \in U$ :

1. Let $u$ be the subset-vertex associated with $U_{i}=\left\{e_{i_{1}}, e_{i_{2}}, \ldots, e_{i_{\left|U_{i}\right|}}\right\}$.

2. Determine $E(u)$, the set of element-vertices associated with $e_{i_{j}}, j \in 1$.. $\left|U_{i}\right|$.

3. Form $E \leftarrow E \cup\{(u, v) \mid v \in E(u)\}$.

4. Determine $I(u)$, the set of subset-vertices associated with the subset elements in $U$ that intersect with $U_{i}$.

5. Form $E \leftarrow E \cup\{(u, w) \mid w \in I(u), w \neq u\}$.

Note that the only vertices in $V$ that have out-degree $>0$ are the subset-vertices. Additionally, the in-degree of each position-vertex is no more than $k$. Clearly, any set $U^{\prime} \in U$ is a set cover of $S$ if and only if the set $V^{\prime}$ is a semi-cover of $G$, where $V^{\prime}$ is the set of subset-vertices associated with the subsets in $U^{\prime}$. 
Corollary 1 For the $k$-bounded relaxed vertex cover problem $\left(R V C P_{k}\right)$ there is a polynomial time algorithm with an approximation ratio $k-\frac{(k-1) \cdot \ln \ln n}{\ln n}$, where $n=|E|$.

This follows directly from Theorem 1 and the results obtained in [10].

\section{$3 \quad \mathbf{R V C P}_{k}$ and the $k$-Covers Problem}

Here we consider the decision form of the $k$-covers problem: given a string $\boldsymbol{x}$ and integers $k>1$ and $\nu$, decide whether there exists a $k$-cover of $\boldsymbol{x}$ of cardinality $\nu$. We call this problem $k \boldsymbol{C P}$ and we show that it is a special case of $\mathrm{RVCP}_{k}$.

Theorem 2 Every instance of $k C P$ can be reduced to an instance of $R V C P_{k}$ in polynomial time.

Proof: Suppose now that a string $\boldsymbol{x}=\boldsymbol{x}[1 . . n]$ and an integer $k$ are given. Let $n$ be the length of the string $\boldsymbol{x}$ and $n^{\prime}$ be the number of distinct $k$-substrings (substrings of length $k)$ in $\boldsymbol{x}$. We initialize a directed graph $G_{\boldsymbol{x}, k}=(V, E)$, where $|V|=n^{\prime}+n$ and $E=\emptyset$. We called the first $n^{\prime}$ vertices in $V$ the $k$-substring-vertices and the remaining $n$ vertices the position-vertices. For every distinct $k$-substring $u_{i}$ where $i=1, \ldots, n^{\prime}$, compute

1. The set $P\left(u_{i}\right)$ of position-vertices that correspond to the positions in $\boldsymbol{x}$ that can be covered by $u_{i}$, where a position $i$ can be covered by $u_{i}$ if and only if $u_{i}$ occurs at some position $j \in i-k+1 . . i$.

2. The set $O\left(u_{i}\right)$ of $k$-substring-vertices that correspond to all $k$-substrings of $\boldsymbol{x}$ that overlap with $u_{i}$, where two strings overlap if and only if there is a non empty prefix of one of them which equals a suffix of the other.

3. If $u$ is the $k$-substring-vertex related to $u_{i}$ then $E$ is updated as follows:

$$
E \leftarrow E \cup\left\{(u, v) \mid v \in P\left(u_{i}\right)\right\} \cup\left\{(u, w) \mid w \in Q\left(u_{i}\right), w \neq u\right\} .
$$

Clearly, the $k$-substring-vertices are the only vertices with out-degree $>0$. Accordingly, any vertex semi-cover of $G_{\boldsymbol{x}, k}$ is a set of $k$-substring-vertices. Note that each position in $\boldsymbol{x}$ cannot be covered with more than $k$ distinct $k$-substrings. Thus, the in-degree of all position-vertices is no more than $k$.

Consider a vertex semi-cover $V^{\prime}$ of $G_{\boldsymbol{x}, k}$. Let vertex $s$ be one of the vertices in $V^{\prime}$ and let $u_{s}$ be the $k$-substring corresponding to $s$. Then in addition to the outgoing and incoming arcs of $s$, all the arcs pointed to each position-vertex $v \in P\left(u_{s}\right)$ will be semicovered according to condition (C3). This is because the sources of these arcs are $k$ substring-vertices $\in O\left(u_{s}\right)$

If the alphabet of $\boldsymbol{x}$ is ordered, an algorithm to compute $G_{\boldsymbol{x}, k}$ from $\boldsymbol{x}$ can be implemented in $O(n \log n)$ time using a straightforward approach, somewhat faster using a suffix tree to sort the $k$-strings.

For example, if $\boldsymbol{x}=a a b b a b$ and $k=2$, then the only four distinct $k$-substrings are $a a, a b, b a$, and $b b$. Let $s_{1}, s_{2}, s_{3}, s_{4}$ be the $k$-substring-vertices associated with them. The corresponding graph $G_{a a b b a b, 2}$ is: 


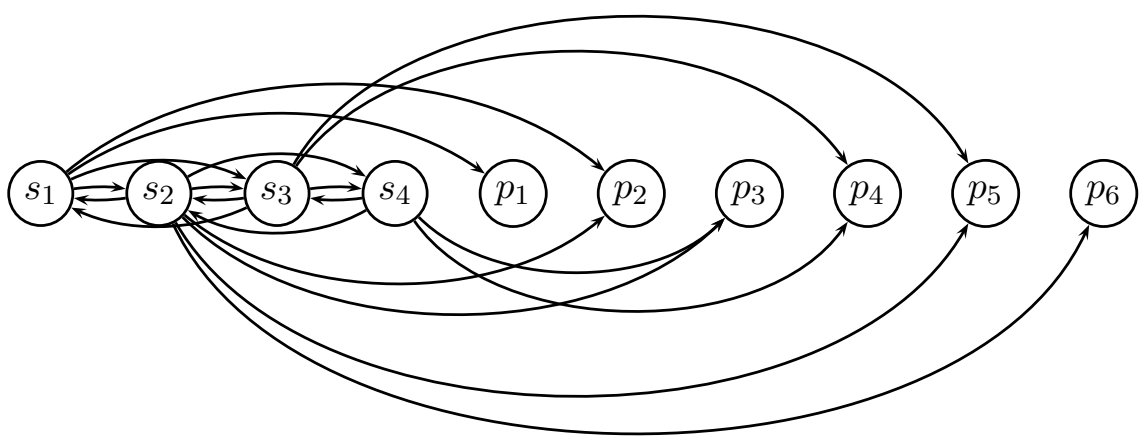

where each position-vertex $p_{i}$ represents position $i$ in $\boldsymbol{x}$. The sets $V_{1}^{\prime}=\left\{s_{1}, s_{2}, s_{3}\right\}$ and $V_{2}^{\prime}=\left\{s_{1}, s_{2}, s_{4}\right\}$ are semi-covers of $G_{a a b b a b, 2}$. The semi-cover $V_{1}^{\prime}$ corresponds to the minimum $k$-cover $U_{1}=\{a a, a b, b a\}$ while $V_{2}^{\prime}$ corresponds to $U_{2}=\{a a, a b, b b\}$.

Theorem 2 and Corollary 1 show that there is an approximation algorithm that calculates a minimum $k$-cover of a given string $\boldsymbol{x}$ whose cardinality is at most $k-\frac{(k-1) \cdot \ln \ln 2 k n}{\ln 2 k n}$ times the minimum. This is because the number of arcs in graph $G_{\boldsymbol{x}, k}$ formed from $\boldsymbol{x}=\boldsymbol{x}[1 . . n]$ is at most $2 \mathrm{kn}$.

\section{Open Problems}

We have shown that for $k \geq 2$, the $k$-covers problem $k \mathrm{CP}$ is equivalent to $\mathrm{RVCP}_{k}$, hence that efficient algorithms can be used to approximate a minimum $k$-cover as specified in Section 3. Interesting questions remain:

(Q1) The set $\mathcal{G}$ of graphs $G_{\boldsymbol{x}, k}$ in some sense describes the structure of all strings. To our knowledge these graphs have not previously been reported in the literature. Can the graphs of $\mathcal{G}$ be characterized in another way? What are their defining properties?

(Q2) The NP-completeness proof given in [6] is based upon strings whose length $n$ is a function of three parameters: $k$ (the length of the covering substrings), $r$ (the number of variables in the corresponding 3-SAT problem), and $s$ (the number of clauses in the corresponding 3-SAT problem). A short calculation shows that in fact

$$
n=(18 k+7) r+(42 k-3) s+(2 k-1),
$$

while at the same time the minimum cover size

$$
\nu=9 r+6 r^{\prime}+8 s+1, r^{\prime} \leq r .
$$

Let us call the ratio $\gamma_{k}=n /(\nu k)$ the $k$-coverability of the string $\boldsymbol{x}[1 . . n]$; observe that $\gamma_{k}$ has as an upper bound the average number of occurrences in $\boldsymbol{x}$ of the strings in the minimum $k$-cover. Since $\nu \leq 15 r+8 s+1$, we see then that for the class of strings constructed in [6], $\gamma_{k}>6 / 5$; in other words, the strings in the $k$-cover occur on average somewhat frequently in $\boldsymbol{x}$. What happens when $\gamma_{k} \leq 6 / 5$ ? Can we find a polynomial-time algorithm to compute a minimum $k$-cover given that $\gamma_{k}$ falls below 
a certain threshold? For "most" strings and some sufficiently large $k$, we expect that $\nu=\lceil n / k\rceil$, so that $\gamma_{k} \approx 1$; thus such an algorithm would in fact handle most of the cases that arise.

\section{Acknowledgements}

Costas S. Iliopoulos was supported in part by a Marie Curie fellowship, Wellcome \& Royal Society grants. Manal Mohamed was supported by an EPSRC studentship. W.F. Smyth was supported in part by a grant from the Natural Sciences \& Engineering Research Council of Canada.

\section{References}

[1] Alfred V. Aho, John E. Hopcroft and Jeffey D. Ullman, The Design \& Analysis of Computer Algorithms, Addison-Wesley, 1974.

[2] A. Apostolico, M. Farach and C. S. Iliopoulos, Optimal Superprimitivity Testing for Strings, Inform. Process. Lett. 39, 1991, 17-20.

[3] Alberto Apostolico and Franco P. Preparata, Optimal Off-Line Detection of Repetitions in a String, Theoret. Comput. Sci. 22, 1983, 297-315.

[4] Bar-Yehuda and S. Even, A Linear Time Approximation Algorithm for the Weighted Vertex Cover Problem, Journal of Algorithms 2, 1981, 198-203.

[5] Gerth S. Brodal, Rune B. Lyngsø, Christian N. S. Pedersen and Jens Stoye, Finding Maximal Pairs with Bounded Gap, J. Discrete Algs. 1, 2000, 77-103.

[6] Richard Cole, Costas S. Iliopoulos, Manal Mohamed, W. F. Smyth and Lu Yang, Computing the Minimum k-Cover of a String, Proc. Prague Stringology Conf.'03, 2003, 51-62.

[7] Maxime Crochemore, An Optimal Algorithm for Computing All the Repetitions in a Word, Inform. Process. Lett. 12-5, 1981, 244-248.

[8] František Franěk, W. F. Smyth and Yudong Tang, Computing all Repeats Using Suffix Arrays, J. Automata, Languages \& Combinatorics 8-4, 2003, 579-591.

[9] Michael R. Garey and David S. Johnson, Computers and Intractability: a Guide to the Theory of NP-Completeness, Freeman, 1979.

[10] E. Halperin, Improved Approximation Algorithms for the Vertex Cover Problem in Graphs and Hypergraph, SIAM Journal on Computing 31(5), 2002, 1608-1623.

[11] D.S. Hochbaum, Approximation Algorithms for the Set Covering and Vertex Cover Problems, SIAM Journal on Computing 11(3), 1982, 555-556.

[12] Costas S. Iliopoulos and W. F. Smyth, On-Line Algorithms for k-Covering, Proc. Ninth Australasian Workshop on Combinatorial Algorithms, 1998, 107-116.

[13] Roman Kolpakov and Gregory Kucherov, On Maximal Repetitions in Words, J. Discrete Algs. 1, 2000, 159-186.

[14] Yin Li and W. F. Smyth, Computing the Cover Array in Linear Time, Algorithmica 32-1, 2002, 95-106.

[15] Michael G. Main, Detecting Leftmost Maximal Periodicities, Discrete Applied Maths. 25, 1989, $145-153$.

[16] Michael G. Main and Richard J. Lorentz, An $O(n \log n)$ Algorithm for Finding All Repetitions in a String, J. Algs. 5, 1984, 422-432. 
[17] Jeanette P. Schmidt, All Highest Scoring Paths in Weighted Grid Graphs and their Application to Finding All Approximate Repeats in Strings, SIAM J. Comput. 27-4, 1998, 972-992.

[18] Lu Yang, Computing the Minimum k-Cover of a String, M. Sc. thesis, McMaster University, 2000, 86 pp. 